\title{
Lower Plasma Creatinine and Urine Albumin in Individuals at Increased Risk of Type 2 Diabetes with Factor V Leiden Mutation
}

\author{
Andreas Peter, ${ }^{1,2,3}$ Andreas Fritsche, ${ }^{1,2,3}$ Fausto Machicao, ${ }^{1,2,3}$ \\ Peter P. Nawroth, ${ }^{3,4}$ Hans-Ulrich Häring, ${ }^{1,2,3}$ and Berend Isermann ${ }^{5}$ \\ ${ }^{1}$ Department of Internal Medicine, Division of Endocrinology, Diabetology, Angiology, Nephrology and Clinical Chemistry, \\ Eberhard-Karls University Tübingen, Otfried-Müller-Straße 10, 72076 Tübingen, Germany \\ ${ }^{2}$ Institute for Diabetes Research and Metabolic Diseases of the Helmholtz Center Munich, \\ University of Tübingen, 72076 Tübingen, Germany \\ ${ }^{3}$ German Center for Diabetes Research (DZD), 85764 Neuherberg, Germany \\ ${ }^{4}$ Department of Internal Medicine I and Clinical Chemistry, University of Heidelberg, \\ Im Neuenheimer Feld 410, 69120 Heidelberg, Germany \\ ${ }^{5}$ Department of Clinical Chemistry and Pathobiochemistry, Otto-von-Guericke-University Magdeburg, \\ Leipziger Straße 44, 39120 Magdeburg, Germany
}

Correspondence should be addressed to Andreas Peter; andreas.peter@med.uni-tuebingen.de

Received 23 November 2013; Accepted 23 December 2013; Published 4 March 2014

Academic Editors: C.-H. Anderwald and J. Vrbikova

Copyright (C) 2014 Andreas Peter et al. This is an open access article distributed under the Creative Commons Attribution License, which permits unrestricted use, distribution, and reproduction in any medium, provided the original work is properly cited.

\begin{abstract}
The factor V Leiden (FVL) mutation is the most frequent genetic cause of venous thrombosis in Caucasians. However, protective effects have been suggested to balance the disadvantages. We have recently observed protective effects of FVL mutation on experimental diabetic nephropathy in mice as well as an association with reduced albuminuria in two human cohorts of diabetic patients. In the present study we aimed to reevaluate these findings in an independent, larger cohort of 1905 Caucasians at risk of developing type 2 diabetes and extend possible associations to earlier disease stages of nephropathy. Carriers of FVL mutation had a significantly lower urine albumin excretion $(P=0.03)$ and tended to have lower plasma creatinine concentrations $(P=0.07)$. The difference in plasma creatinine concentrations was significant after adjustment for the influencing factors: age, gender, and lean body mass $(P=0.048)$. These observations at a very early "disease" stage are an important extension of previous findings and suggest that modification of glomerular dysfunction by FVL mutation is relevant during very early stages of diabetic nephropathy. This makes the underlying mechanism an interesting therapeutic target and raises the question whether FVL mutation may also exert protective effects in other glomerulopathies.
\end{abstract}

\section{Introduction}

The factor V Leiden (FVL) mutation is the most frequent genetic cause of venous thrombosis in Caucasians. The high frequency of the FVL mutation implies associated positive effects which balance the negative sequels of the FVL mutation [1]. Indeed, several protective effects associated with the FVL mutation and potentially conveying a positive selection bias have been proposed [2]. Thus, the presence of the FVL mutation has been associated with lower peripartal blood loss and reduced mortality in sepsis $[2,3]$. Obviously the amelioration of these acute and potentially deadly health threats mediates a positive selection pressure, maintaining the FVL mutation in the gene pool despite the potentially negative effects associated with venous thrombosis. We have recently proposed that the protective mechanism(s) selected in FVL carriers may not only be relevant in acute diseases, but also modulate the course of chronic diseases, which pose the prevailing health threats in modern times. Thus, low but sustained thrombin generation associated with the 
TABLE 1: Participant characteristics of the total cohort and those subjects with available urine samples are displayed.

\begin{tabular}{|c|c|c|c|c|c|c|}
\hline \multirow{2}{*}{ Characteristic } & \multicolumn{2}{|c|}{ All subjects } & \multirow{2}{*}{$P$} & \multicolumn{2}{|c|}{ Subjects with urine samples } & \multirow{2}{*}{$P$} \\
\hline & Wildtype & FVL mutation & & Wildtype & FVL mutation & \\
\hline$N$ & 1780 & 125 & - & 744 & 56 & - \\
\hline Gender (females/males) & $1171 / 609$ & $84 / 41$ & $0.75^{*}$ & $481 / 263$ & $41 / 15$ & $0.19^{*}$ \\
\hline Age (years) & $39.7 \pm 0.3$ & $41.1 \pm 1.2$ & 0.26 & $43.7 \pm 0.5$ & $44.4 \pm 1.7$ & 0.72 \\
\hline Diabetes & $99(5.6 \%)$ & $6(4.8 \%)$ & $0.71^{*}$ & $57(7.7 \%)$ & $3(5.4 \%)$ & $0.53^{*}$ \\
\hline Body weight (kg) & $87.7 \pm 0.6$ & $85.7 \pm 2.4$ & 0.41 & $99.3 \pm 1.12$ & $95.6 \pm 4.10$ & 0.38 \\
\hline $\mathrm{BMI}\left(\mathrm{kg} \cdot \mathrm{m}^{-2}\right)$ & $30.17 \pm 0.21$ & $29.73 \pm 0.81$ & 0.61 & $34.30 \pm 0.37$ & $33.36 \pm 1.37$ & 0.50 \\
\hline Lean body mass (kg) & $57.02 \pm 0.33$ & $55.63 \pm 1.27$ & 0.29 & $60.09 \pm 0.60$ & $57.84 \pm 2.19$ & 0.32 \\
\hline $\operatorname{HbAlc}(\%)$ & $5.42 \pm 0.01$ & $5.42 \pm 0.05$ & 0.88 & $5.69 \pm 0.02$ & $5.59 \pm 0.09$ & 0.26 \\
\hline Blood pressure syst. (mmHg) & $123.9 \pm 0.49$ & $126.4 \pm 1.84$ & 0.19 & $125.8 \pm 0.6$ & $125.9 \pm 2.18$ & 0.94 \\
\hline Blood pressure diast. (mmHg) & $77.8 \pm 0.33$ & $78.6 \pm 1.24$ & 0.55 & $79.4 \pm 0.4$ & $79.5 \pm 1.44$ & 0.96 \\
\hline Insulin sensitivity & $14.94 \pm 0.25$ & $14.64 \pm 0.95$ & 0.76 & $11.48 \pm 0.34$ & $11.87 \pm 1.29$ & 0.77 \\
\hline Fasting glucose (mmol/L) & $5.20 \pm 0.02$ & $5.26 \pm 0.06$ & 0.34 & $5.39 \pm 0.02$ & $5.27 \pm 0.09$ & 0.19 \\
\hline Plasma creatinine (mg/dL) & $0.849 \pm 0.004$ & $0.822 \pm 0.014$ & 0.07 & $0.856 \pm 0.006$ & $0.825 \pm 0.024$ & 0.24 \\
\hline Urine albumin $(\mathrm{mg} / \mathrm{L})$ & - & - & - & $31.3 \pm 7.6$ & $10.7 \pm 27.6$ & 0.03 \\
\hline Plasma creatinine $(\mathrm{mg} / \mathrm{dL})^{* *}$ & $0.849 \pm 0.003$ & $0.823 \pm 0.013$ & $0.048^{* *}$ & - & - & - \\
\hline
\end{tabular}

Values represent means \pm SE (standard error). For statistical analyses, nonnormally distributed parameters were log transformed. ISI: insulin sensitivity index. Significant differences are marked by bold font.

${ }^{*}$ Chi-square test; ${ }^{* *}$ adjusted for age, gender, and lean body mass.

presence of the FVL mutation results in larger but more stable plaques in atherosclerosis prone mice, indicating a partial benefit [4]. Furthermore, in a second mouse model we observed a protective effect of the FVL mutation on experimental diabetic nephropathy [5]. The potential mechanisms include prevention of glomerular endothelial cell and podocyte dysfunction as well as reduction of extracellular matrix accumulation [5]. These results were supported by an association of FVL mutation with reduced albuminuria in two human cohorts of 200 type 1 and 350 type 2 diabetic patients [5]. In the present study we aimed to reevaluate these findings in an independent, larger cohort and extend the possible associations to earlier disease stages of nephropathy.

\section{Materials and Methods}

We investigated 1905 Caucasians who participated in ongoing studies on the pathogenesis of type 2 diabetes [6]. A written consent was obtained from all participants and the local ethics committee approved the study protocol. Unlike in the previous report, which included patients with established diabetes mellitus, inclusion criterion for the current study was an elevated risk of developing type 2 diabetes, that is, family history of type 2 diabetes, body mass index (BMI) $>27 \mathrm{~kg} / \mathrm{m}^{2}$, diagnosis of impaired glucose tolerance, or previous diagnosis of gestational diabetes. All participants underwent an oral glucose tolerance test (OGTT). After an overnight fast, $75 \mathrm{~g}$ glucose was ingested at 8:00 a.m.; plasma glucose and insulin concentrations were determined after 0 , $30,60,90$, and $120 \mathrm{~min}$. Insulin sensitivity was assessed from glucose and insulin values during the five-point OGTT with the Matsuda index [7]. In 800 subjects urine samples were available for albumin measurements.
The HPLC method (Tosoh Alc 2.2 HLC-723, Tokyo, Japan, and Tosoh G8 HPLC Alc analyzer) was used for $\mathrm{HbAlc}$ measurements, plasma glucose was determined using a bedside glucose analyzer (glucose-oxidase method; YSI, Yellow Springs Instruments), urine albumin was determined on the BN Prospec nephelometer, plasma creatinine was measured enzymatically on the ADVIA 1800 clinical chemistry analyzer, and insulin was analyzed using the ADVIA Centaur XP immunoassay system (all from Siemens Healthcare Diagnostics, Eschborn, Germany). Lean body mass was measured by bioelectrical impedance (BIA-101; RJL Systems, Detroit). The Sequenom MassARRAY platform (Sequenom, San Diego) was used to genotype for FVL mutation (rs6025). For statistical analyses nonnormally distributed parameters were $\log$ transformed. Statistical analyses were conducted with JMP 8.0 (SAS Institute, Cary, NC).

\section{Results and Discussion}

Participant characteristics are supplied in Table 1. Based on a $75 \mathrm{~g}$ oral glucose tolerance test and/or HbAlc measurements performed at inclusion, 105 subjects were newly diagnosed with diabetes according to the criteria of the American Diabetes Association [8]. The frequency of newly diagnosed diabetes mellitus did not differ between FVL-negative and -positive individuals. To evaluate the impact of the FVL mutation on albuminuria and plasma creatinine a dominant model was used since only one homozygous FVL carrier was identified.

FVL carriers showed no significant differences in metabolic traits or blood pressure (Table 1). Carriers of FVL mutation had a significantly lower urine albumin excretion $(P=$ 0.03 ) (Table 1). Even when the subjects with newly diagnosed diabetes were excluded, this trend was still present $(P=$ 
0.050). We next analyzed whether the FVL mutation also influences plasma creatinine levels. Carriers of FVL mutation tended to have lower plasma creatinine concentrations $(P=$ 0.07 ). The difference in plasma creatinine concentrations was significant after adjustment for the influencing factors: age, gender, and lean body mass $(P=0.048)$. This association remained unchanged after exclusion of subjects with newly diagnosed diabetes $(P=0.048)$. The lower urine albumin excretion in individuals with an increased risk to develop type 2 diabetes underscores that FVL mutation conveys a protective effect in regard to urine albumin excretion, an established marker of glomerular dysfunction. In addition to lower urine albumin excretion we observed lower plasma creatinine values in FVL-positive individuals. Of note, a retrospective analysis of our previously published cohort of type 2 diabetic patients [5] revealed a similar trend for lower plasma creatinine levels (mean $1.10 \mathrm{mg} / \mathrm{dl}$ in FVLnegative patients versus $0.89 \mathrm{mg} / \mathrm{dl}$ in FVL-positive patients, $P=0.082)$. The impact of the FVL mutation appears to differ in regard to urine albumin excretion and plasma creatinine concentrations, considering that the difference in plasma creatinine concentrations was only of borderline significance or showed a trend between FVL-negative and FVLpositive individuals, while urine albumin excretion differed significantly in these two studies. However, the differences in plasma creatinine concentrations may be less obvious, since albuminuria is an early manifestation of diabetic nephropathy, preceding the rise in the plasma creatinine and as renal impairment will not progress in all patients with albuminuria to more advanced stages hallmarked by increased creatinine plasma concentrations [9]. The future evaluation of a larger number of diabetic patients with advanced stages of diabetic nephropathy may provide more robust results in regard to the effect of the FVL mutation on plasma creatinine concentration in type 2 diabetic patients.

Intriguingly, in the present study we even observed a lower urine albumin excretion as well as plasma creatinine concentrations in nondiabetic individuals at risk of Developing type 2 diabetes and hence at a very early "disease" stage. This is an important extension of previous findings and indicates that the underlying mechanism through which the FVL mutation modifies glomerular dysfunction is of functional relevance during very early stages of diabetic nephropathy, making the underlying mechanism an interesting therapeutic target. Hence, further studies evaluating the nephroprotective mechanism associated with the FVL mutation in diabetic patients are warranted. In addition, these studies raise the question whether the FVL mutation may exert protective effect in other glomerulopathies. Providing answers to these questions may identify novel therapeutic targets for diabetic nephropathy and potentially other glomerular diseases.

\section{Acknowledgments}

This work was supported by grants of the Deutsche Forschungsgemeinschaft (IS 67/2-4), the Stiftung Pathobiochemie, the EFSD (European Foundation for the Study of Diabetes), and the DDS (Deutsche Diabetes Stiftung) to Berend Isermann and a grant from the Dietmar Hopp Stiftung to Berend Isermann and Peter P. Nawroth. This study was supported in part by a Grant (01GI0925) from the German Federal Ministry of Education and Research (BMBF) to the German Center for Diabetes Research (DZD e.V.). Peter P. Nawroth is an associated partner in the German Centre for Diabetes Research (DZD).

\section{References}

[1] P. W. Majerus, “Bad blood by mutation," Nature, vol. 369, no. 6475, pp. 14-15, 1994.

[2] P. G. Lindqvist and B. Dahlbäck, "Carriership of factor V leiden and evolutionary selection advantage," Current Medicinal Chemistry, vol. 15, no. 15, pp. 1541-1544, 2008.

[3] B. A. Kerlin, S. B. Yan, B. H. Isermann et al., "Survival advantage associated with heterozygous factor $\mathrm{V}$ Leiden mutation in patients with severe sepsis and in mouse endotoxemia," Blood, vol. 102, no. 9, pp. 3085-3092, 2003.

[4] S. Seehaus, K. Shahzad, M. Kashif et al., "Hypercoagulability inhibits monocyte transendothelial migration through protease-activated receptor-1-, phospholipase-c $\beta$-, phosphoinositide 3-kinase-, and nitric oxide-dependent signaling in monocytes and promotes plaque stability," Circulation, vol. 120, no. 9, pp. 774-784, 2009.

[5] H. Wang, T. Madhusudhan, T. He et al., "Low but sustained coagulation activation ameliorates glucose-induced podocyte apoptosis: protective effect of factor $\mathrm{V}$ Leiden in diabetic nephropathy," Blood, vol. 117, no. 19, pp. 5231-5242, 2011.

[6] A. Peter, A. Fritsche, N. Stefan, M. Heni, H.-U. Häring, and E. Schleicher, "Diagnostic value of hemoglobin alc for type 2 diabetes mellitus in a population at risk," Experimental and Clinical Endocrinology and Diabetes, vol. 119, no. 4, pp. 234-237, 2011.

[7] M. Matsuda and R. A. DeFronzo, "Insulin sensitivity indices obtained from oral glucose tolerance testing: comparison with the euglycemic insulin clamp," Diabetes Care, vol. 22, no. 9, pp. 1462-1470, 1999.

[8] American Diabetes Association, "Diagnosis and classification of diabetes mellitus," Diabetes Care, vol. 33, supplement 1, pp. S62-S69, 2010.

[9] P. P. Nawroth and B. Isermann, "Mechanisms of diabetic nephropathy-old buddies and newcomers part 1," Experimental and Clinical Endocrinology and Diabetes, vol. 118, no. 9, pp. 571-576, 2010.

\section{Conflict of Interests}

The authors state that they have no conflict of interests. 


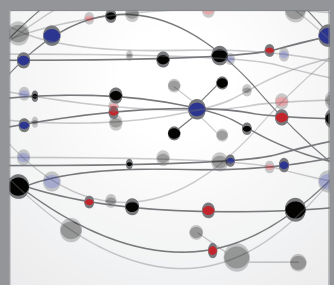

The Scientific World Journal
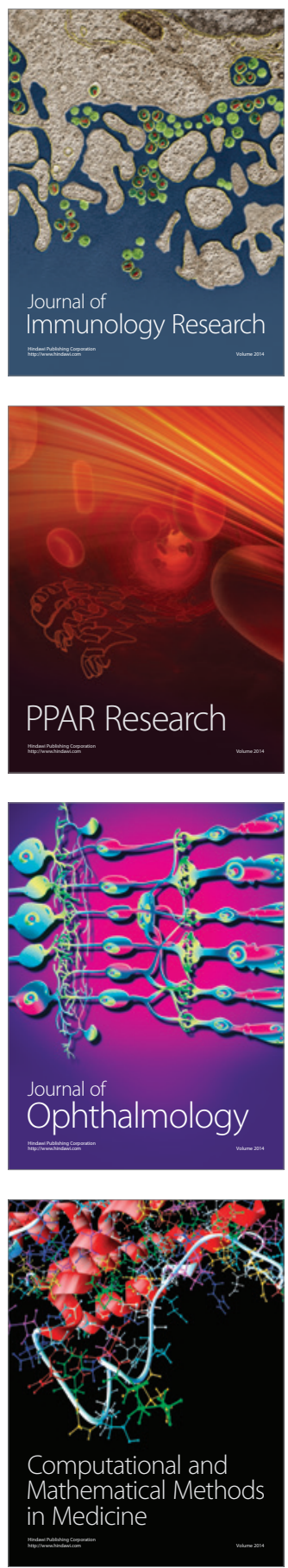

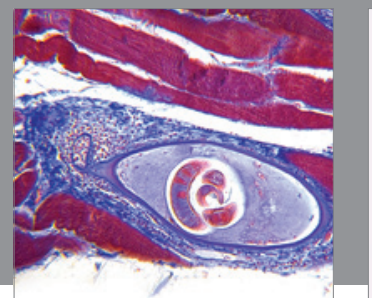

Gastroenterology

Research and Practice
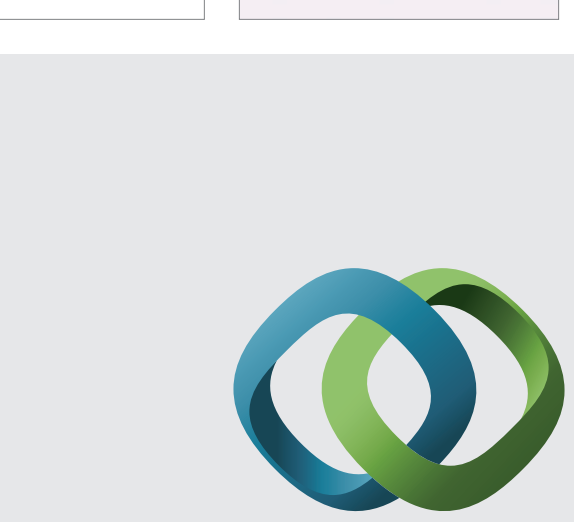

\section{Hindawi}

Submit your manuscripts at

http://www.hindawi.com
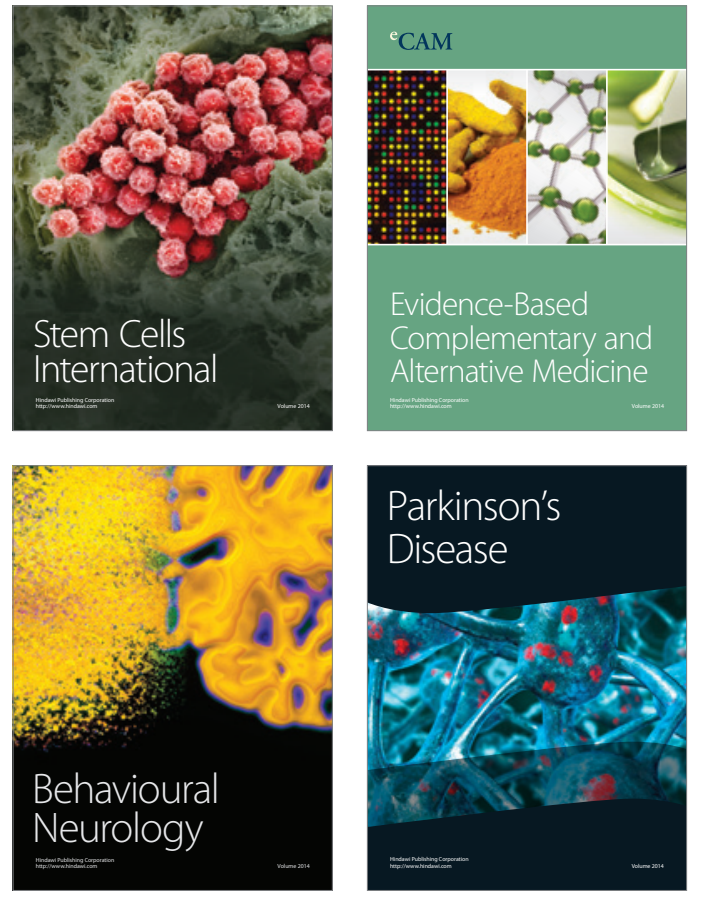
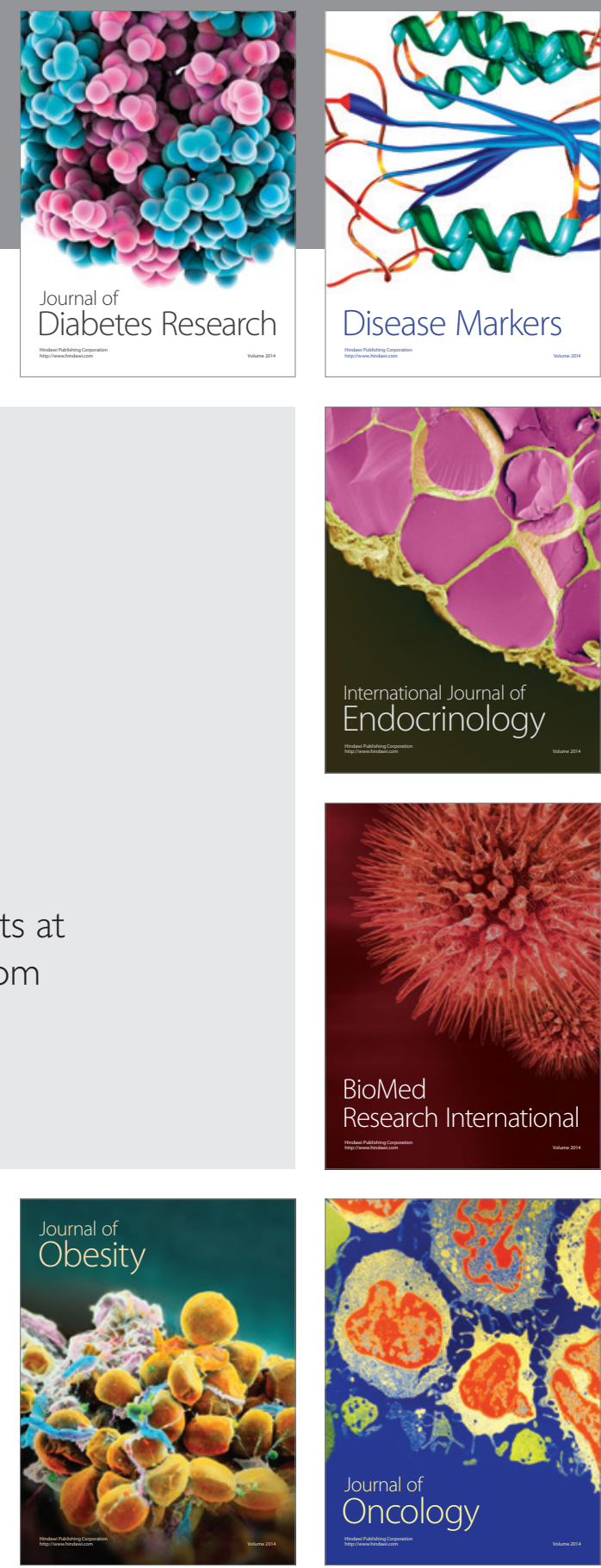

Disease Markers
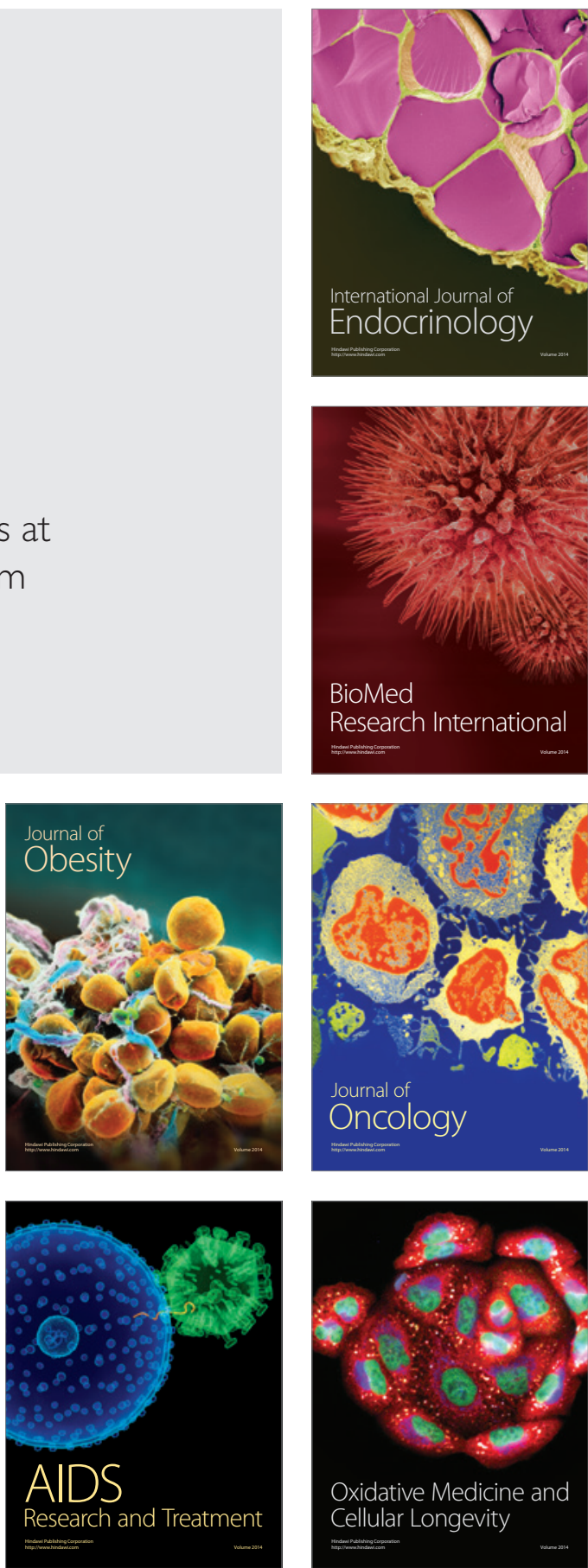